\title{
Comparación entre dexmedetomidina en infusión intravenosa vs. lidocaína en infusión intravenosa para el control de dolor refractario a tratamiento opioide en pacientes de cuidados paliativos
}

\author{
O. Carrillo Torres, M. M. Gallegos Allier y M. Jiménez Olvera \\ Servicio Clínica del Dolor. Hospital General de México O.D.
}

Carrillo Torres O, Gallegos Allier MM, Jiménez Olvera $M$. Comparación entre dexmedetomidina en infusión intravenosa vs. lidocaína en infusión intravenosa para el control de dolor refractario a tratamiento opioide en pacientes de cuidados paliativos. Rev Soc Esp Dolor 2015; 22(1): 7-12.

\begin{abstract}
Introduction: Refractory pain is defined as that which remains persistent (EVA 6 or more) despite treatment with opioids (with 1 or more previous rotations) + anti-inflammatory drugs (NSAIDs or corticosteroids). Alpha-2 agonists and/or local anesthetics have been used for treating. Analgesic response to the administration of dexmedetomidine appears to occur at the level of dorsal root neuron, where the alpha- 2 agonists block the release of substance $\mathrm{P}$ in the nociceptive pathway. The central analgesic effect is mediated by the activation of descending inhibitory pathways, by blocking the receptors of aspartate and glutamate. The use of intravenous lidocaine can suppress ectopic neural discharges primary afferents from injured due to its blocking voltage-dependent sodium channels. Furthermore it has been demonstrated activation of endogenous opioid system lidocaine infusion systemically.

Objective: To evaluate the analgesic effect of intravenous dexmedetomidine infusion compared with intravenous lidocaine infusion for pain refractory to opioids in palliative care patients.

Material and methods: This is a randomized, triple-blind study with consecutive sampling. The analysis of results with measures of central tendency and dispersion. To compare quantitative variables $\mathrm{T}$ test was used for independent samples. Contingency tables were constructed and graphics through SPSS version 17. Study population: Patients with refractory pain,
\end{abstract}

Recibido: 05-08-13.

Aceptado: 15-12-13. hospitalized within the Palliative Care Program randomly assigned to one of 2 groups whose sample number $(n=14)$ was obtained by difference formula proportions.

Results: For both infusions analgesia with no significant differences. Of the 16 patients, $18 \%(n=3)$ required rescue therapy during infusion and $18 \%(n=3)$ required rescue within 48 hours post-infusion. During infusion of the $42 \%$ lidocaine group need rescue $(\mathrm{n}=3)$ and time within 48 hours post infusion was administered three rescues: $42 \%$ of patients in the lidocaine group ( $\mathrm{n}=2$ ), and $14 \%$ of dexmedetomidine group patients ( $n: 1)$. Regarding sedation during the infusion were reported significant differences ( $p-0.01$ ) increased sedation reporting for dexmedetomidine group. No complications cardiovascular and / or respiratory none of the two groups.

Conclusions: The dexmedetomidine group required less opioid bailouts during infusion and after it. Sedation was greater in the group of dexmedetomidine without presence of cardiovascular and/or respiratory during or after the infusion.

Key words: Refractory pain. Dexmedetomidine. Lidocaine. Palliative care.

\section{RESUMEN}

Introducción: se define dolor refractario como aquel que se mantiene persistente (EVA 6 o más) a pesar de tratamiento con opioides (con 1 o más rotaciones previas) + antiinflamatorios (AINE o corticoides). Para tratamiento se han utilizado agonistas alfa-2 y/o anestésicos locales. La respuesta analgésica a la administración de dexmedetomidina parece producirse a nivel de la neurona de la raíz dorsal, donde los agonistas alfa-2 bloquean la liberación de la sustancia $\mathrm{P}$ en la vía nociceptiva. El efecto analgésico central está mediado por la activación de las vías inhibitorias descendentes gracias al bloqueo de los receptores del aspartato y glutamato. La utilización de lidocaína intravenosa puede suprimir descargas neurales ectópicas procedentes de fibras aferentes primarias lesionadas debido a sus propiedades de bloqueo dependiente de canales de voltaje de sodio. 
Además se ha evidenciado la activación endógena del sistema de opioides por infusión de lidocaína por vía sistémica.

Objetivo: evaluar el efecto analgésico de la infusión de dexmedetomidina intravenosa comparada con infusión de lidocaína intravenosa para dolor refractario a opioides en pacientes bajo cuidados paliativos.

Material y métodos: se trata de un ensayo clínico aleatorizado, triple ciego, con muestreo consecutivo. El análisis de resultados con medidas de tendencia central y de dispersión. Para comparar variables cuantitativas se usó t de Student para muestras independientes. Se construyeron tablas de contingencia y gráficos a través del programa estadístico SPSS versión 17. Población de estudio: pacientes con dolor refractario, hospitalizados dentro del Programa de Cuidados Paliativos asignados aleatoriamente a uno de los 2 grupos cuyo número de muestra ( $n=14$ ) se obtuvo por fórmula de diferencia de proporciones.

Resultados: en cuanto a la analgesia con ambas infusiones no hubo diferencias significativas. De los 16 pacientes, $18 \%$ (n: 3) requirieron terapia de rescate durante la infusión y $18 \%$ (n: 3) requirieron rescate durante las 48 horas postinfusión. Durante la infusión el 42 \% del grupo de lidocaína necesitó rescate (n: 3) y en el tiempo postinfusión antes de 48 horas se administraron 3 rescates: $42 \%$ de los pacientes del grupo de lidocaína (n: 2) y $14 \%$ de los pacientes del grupo dexmedetomidina (n: 1). En cuanto a sedación durante la infusión se reportaron diferencias significativas (p: -0,01) reportándose mayor sedación en el grupo de dexmedetomidina. No se presentaron complicaciones cardiovasculares y/o respiratorias en ninguno de los dos grupos.

Conclusiones: el grupo de dexmedetomidina requirió menos rescates con opioides durante la infusión y posterior a ella. Se presentó mayor sedación en el grupo de dexmedetomidina sin presencia de complicaciones cardiovasculares y/o respiratorias durante o posterior a la infusión.

Palabras clave: Dolor refractario. Dexmedetomidina. Lidocaína. Cuidados paliativos.

\section{INTRODUCCIÓN}

La OMS define cuidados paliativos como el «enfoque que mejora la calidad de vida de pacientes y familias que se enfrentan a problemas asociados con enfermedades amenazantes para la vida, a través de la prevención y alivio del sufrimiento por medio de la identificación temprana e impecable evaluación y tratamiento del dolor y otros problemas, físicos, psicológicos y espirituales» (1).

Es complicado abordar el tema del dolor ya que es difícil desde definirlo hasta clasificarlo. No es una experiencia sensorial discreta entendido como un conjunto definido de estímulos que actúan sobre una vía específica para obtener una sensación en particular, sino más es un conjunto diverso de fenómenos complejos de la percepción que se caracterizan por una naturaleza desagradable o preocupante (2).

Por tanto se trata de una experiencia subjetiva, ya que lesiones similares provocan dolor que puede manifestarse de diversas formas en distintos pacientes (3).
El dolor refractario se define como la ausencia de control analgésico adecuado con opioides potentes a una dosis suficiente que provoque efectos secundarios intolerables a pesar de las mejores medidas para controlarlos (1).

Las alternativas terapéuticas para el manejo del dolor refractario comprenden una cuidadosa revaloración del dolor, así como la adecuación e intensificación de fármacos coadyuvantes, técnicas anestésicas intervencionistas, cambio de vía de administración, u otras terapias como radioterapia analgésica o rotación de opioides (1).

La activación alfa- 2 adrenérgica constituye una parte esencial en la red intrínseca de control del dolor del sistema nervioso central. Se encuentra densamente distribuido en la sustancia gelatinosa del asta dorsal en los humanos y se cree que es un importante sitio de acción en el que se puede aminorar el dolor (4).

Su aplicación en bolos menores a $1 \mu \mathrm{g} / \mathrm{kg}$ o la infusión continua a dosis menores a $2 \mu \mathrm{g} / \mathrm{kg} / \mathrm{hora}$ no parecen inducir efectos hemodinámicos indeseables (5).

La respuesta analgésica a la administración de dexmedetomidina parece producirse a nivel de la neurona de la raíz dorsal, donde los agonistas alfa-2 bloquean la liberación de la sustancia P en la vía nociceptiva. Se considera que estos efectos analgésicos y sedantes aparecen gracias a su mecanismo de acción sobre la proteína G inhibitoria, que incrementa la conductancia a través de los canales del potasio. El efecto analgésico central está mediado por la activación de las vías inhibitorias descendentes, gracias al bloqueo de los receptores del aspartato y glutamato. De esta forma se puede atenuar la hiperexcitabilidad espinal, lo que ejerce un verdadero efecto preventivo del dolor al impedir la estimulación eléctrica repetida (6-9). Han sido aprobados para tratar el dolor neuropático crónico medular, que a su vez se traduce en la liberación de acetilcolina para aliviar el dolor $(10,11)$. Se ha demostrado mejoría a nivel algológico donde otros tratamientos, incluyendo infusión de anestésicos locales, habían fracasado, produciendo disminución de la dosis de opiáceos durante la infusión y hasta 24 horas después aún teniendo una vida media de 2 a 3 horas $(12,13)$.

Entre las ventajas de la utilización de la dexmedetomidina en cuidados paliativos están: la inducción de analgesia de forma rápida, la de ser ahorrador de opiáceos delimitando los efectos intolerables de los mismos a altas dosis y la de producir analgesia cuando otras intervenciones han fracasado (14).

Los anestésicos locales son fármacos que, aplicados en concentración suficiente en su lugar de acción, impiden la conducción de impulsos eléctricos por las membranas del nervio y el músculo de forma transitoria y predecible. Varios estudios han confirmado el efecto de los anestésicos locales i.v. como analgésicos $(15,16)$.

La utilización de lidocaína intravenosa puede suprimir descargas neurales ectópicas procedentes de fibras 
aferentes primarias lesionadas debido a sus propiedades de bloqueo dependiente de canales de voltaje de sodio, además de activación endógena del sistema de opioides (17). Se ha observado una duración variable de efecto analgésico que va desde unas pocas horas a semanas después de una sola infusión i.v., con un potencial de alivio significativamente mayor en relación con el periodo de administración y vida media plasmática $(18,19)$.

\section{OBJETIVO}

Evaluar el efecto analgésico, sedante y los cambios hemodinámicos de la infusión de dexmedetomidina intravenosa comparada contra lidocaína intravenosa para dolor refractario a opioides en pacientes bajo cuidados paliativos.

\section{MATERIAL Y MÉTODOS}

Ensayo clínico aleatorizado que compara a través de escala visual análoga (EVA) la eficacia entre dos tratamientos para el control del dolor refractario. El muestreo fue consecutivo y la asignación de la maniobra en forma aleatoria. Para conseguir el triple ciego ni el paciente ni los médicos (anestesiólogos) que evaluaron la variable dependiente, ni el investigador principal, conocieron el grupo al que pertenece el sujeto de estudio, los cuales fueron muestras representativas de pacientes hospitalizados dentro del Programa de Cuidados Paliativos del Hospital General de México, O.D. durante el año 2012.

\section{Población}

Pacientes de ambos sexos con dolor refractario con algún componente neuropático, hospitalizados dentro del Programa de Cuidados Paliativos en el Hospital General de México, O.D. que cumplieron los criterios de inclusión y exclusión y que aceptaron participar a través de consentimiento informado firmado durante el 2012 asignados aleatoriamente a uno de los 2 grupos cuya $n=14$ para muestra se obtuvo a través de la siguiente fórmula de diferencia de proporciones:

$$
\begin{gathered}
n=\frac{\left[\mathrm{Z}_{1-\alpha} * \sqrt{2 P(1-P)}+\mathrm{z}_{1-\beta} * \sqrt{\mathrm{P}_{1}\left(1-P_{1}\right)+\mathrm{P}_{2}\left(1-P_{2}\right)}\right]^{2}}{\left(P_{1}-P_{2}\right)^{2}} \\
\alpha=0.05 \mathrm{p} 1=0.50 \mathrm{Z} \alpha=1.960 \mathrm{p} 2=0.80 \beta=0.20 \mathrm{p}=0.65 \mathrm{Z} \\
\beta=0.842
\end{gathered}
$$

Se incluyeron pacientes de ambos sexos mayores de 18 años hospitalizados por lo menos 72 horas previas, bajo el programa de cuidados paliativos, con dolor refractario a tratamiento opioide (definido como aquel que, posterior a rotación opioide por fracaso al tratamiento, necesite de 2 o más rescates para control del mismo) que presentara algún componente neuropático y EVA de 6 o más al momento de la evaluación.

\section{Procedimiento}

La asignación aleatoria a uno de los 2 grupos de este estudio fue llevado a cabo de la siguiente manera:

El día de la administración de los fármacos (previa solicitud del servicio tratante) se monitorizó antes y durante la infusión, el trazo ECG, la tensión arterial, la frecuencia cardiaca, la frecuencia respiratoria y la saturación periférica de oxígeno, así como EVA y Ramsay al pie de su cama.

Al grupo I se le aplicó infusión de lidocaína a dosis de $3 \mathrm{mg} / \mathrm{kg}$ de peso ideal diluidos en solución $\mathrm{NaCl}$ 0,9 \% en un volumen total de $100 \mathrm{ml}$ durante 60 minutos, y oxígeno por puntas nasales $3 \mathrm{~lx}$ '.

Al grupo II se le aplicó infusión con dexmedetomidina a una dosis de $0,2 \mathrm{mcg} / \mathrm{kg}$ de peso ideal diluido en solución $\mathrm{NaCl} 0,9 \%$ en un volumen total de $100 \mathrm{ml}$ durante 60 minutos, y oxígenos por puntas nasales 3 lx'.

Se llevó un registro continuo durante 90 minutos de los signos vitales (TA, FC, FR, trazo electrocardiográfico, $\mathrm{SpO}_{2} \%$ ) así como de la escala de Ramsay y la escala visual análoga (EVA) misma explicada previamente para su comprensión por parte del paciente.

Todos los datos fueron registrados 10 minutos previos al inicio de la perfusión correspondiente, continuando durante la misma a $\operatorname{los} 5,10,20,30,40,50,60$ minutos y a $\operatorname{los} 30$ de terminada la infusión y posteriormente a las 2, 3, 6, 12, 24, 36 y 48 horas.

Se consideró exitoso el tratamiento cuando el paciente presentó disminución de EVA por debajo de 6 al final de la infusión, y en caso contrario, se administró rescate analgésico con tramadol entre 20 y $50 \%$ de la dosis prescrita en el tratamiento de base en bolo i.v. sin exceder $400 \mathrm{mg} /$ día, si este fuera el caso, se aplicó buprenorfina subcutánea $150 \mathrm{mcg}$.

La recolección de datos se llevó a cabo en los tiempos antes señalados mediante un formato diseñado especialmente para este fin.

El análisis de resultados se realizó aplicando estadística descriptiva con medidas de tendencia central y de dispersión. La estadística inferencial para la diferencia en EVA entre los grupos se comparó por medio de U de Mann Whitney. Para las variables cuantitativas se usó t de Student para muestras independientes. Se construyeron tablas de contingencia y gráficos a través del programa estadístico SPSS versión 17.

Se elaboró una carta de consentimiento informado en donde se explicaron ampliamente los aspectos que involucran la participación del sujeto en el estudio, el procedi- 
miento terapéutico y la aplicación de la maniobra, así como los beneficios esperados.

\section{RESULTADOS}

Se analizó una muestra de 16 pacientes, de los cuales a 12,5\% (n: 2) no se les evaluó la analgesia postinfusión por presentar EVA al final de la infusión de 6 o más; el 87,5 \% restante (n: 14) concluyó su evaluación total.

Al final del estudió se muestra una diferencia importante entre el número de pacientes en los que fue necesario administrar rescate y los que no, con una diferencia estadísticamente significativa entre el grupo de dexmedetomidina y el grupo de lidocaína (p -0,0001) (Tabla I y Figs. 1-4).

TABLA I. EVIDENCIA DE LA SIGNIFICANCIA A LOS 40 MINUTOS EN LA SEDACIÓN ENTRE LOS 2 GRUPOS ESTUDIADOS

\begin{tabular}{lccc}
\hline \multicolumn{1}{c}{ Ramsay } & Grupo dexmedetomidina & Grupo lidocaína & Valor $p$ \\
\hline Basal & $2,125( \pm 0,35)$ & $2( \pm 0)$ & 0,35 \\
5 minutos & $2,12( \pm 0,35)$ & $2( \pm 0)$ & 0,35 \\
10 minutos & $2,13( \pm 0,35)$ & $2( \pm 0)$ & 0,35 \\
20 minutos & $2,62( \pm 1,06)$ & $2( \pm 0)$ & 0,14 \\
30 minutos & $2,75( \pm 1,03)$ & $2,13( \pm 0,35)$ & 0,08 \\
40 minutos & $3,25( \pm 0,88)$ & $2,37( \pm 0,51)$ & 0,01 \\
50 minutos & $3,625( \pm 0,91)$ & $2,5( \pm 0,53)$ & 0,01 \\
60 minutos & $3,75( \pm 0,88)$ & 0,01 \\
\hline
\end{tabular}

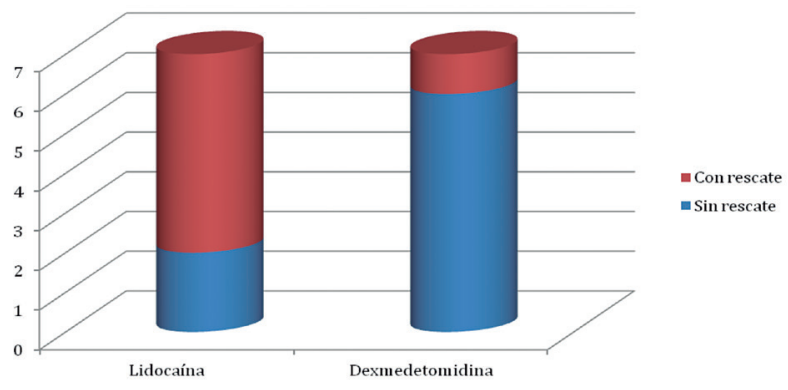

Fig. 1. Diferencia entre el número de pacientes que necesitaron rescate durante el estudio encontrando significancia estadística con $\mathrm{p}-0,001$.

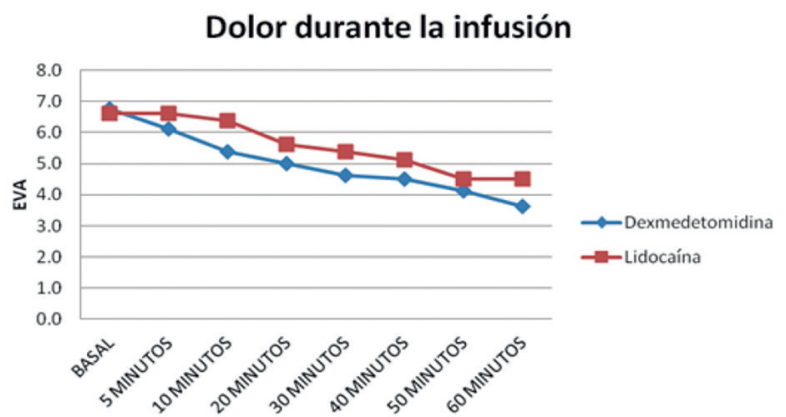

Fig. 2. La diferencia entre el EVA entre el grupo de lidocaína con dexmedetomidina no fue estadísticamente significativo $(\mathrm{p}+0,01)$.

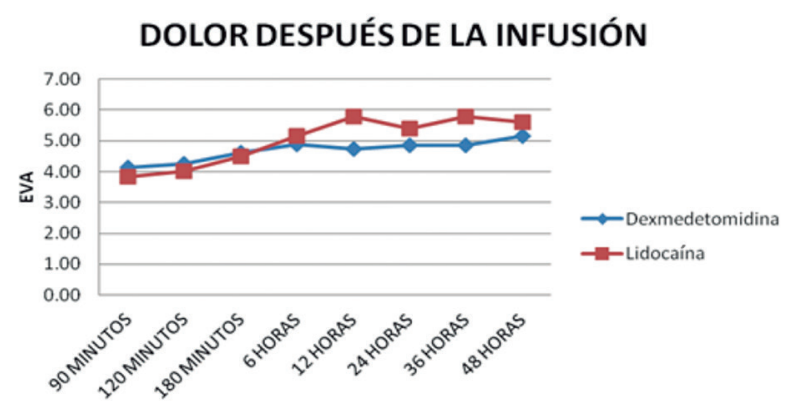

Fig. 3. La diferencia entre el EVA postinfusión entre el grupo de lidocaína comparada con dexmedetomidina no fue estadísticamente significativo $(\mathrm{p}+0,01)$.

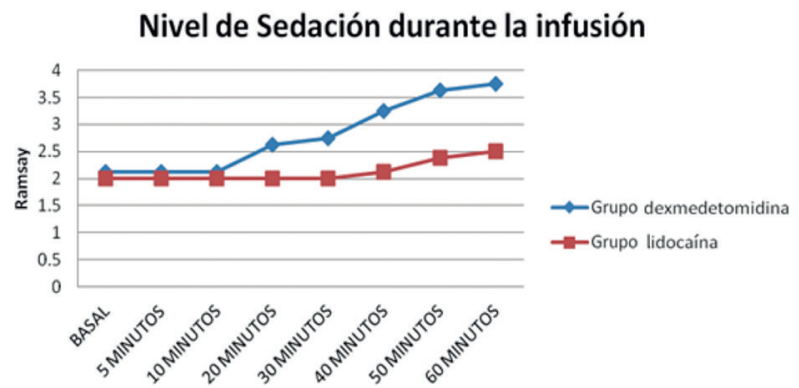

Fig. 4. La diferencia entre la sedación durante la infusión de dexmedetomidina fue estadísticamente significativa desde los 40 minutos. 
Las variables hemodinámicas ( $\mathrm{FC}$, TA, FR y $\left.\mathrm{SaO}_{2}\right)$ no demostraron significancia estadística al realizar la comparación $(\mathrm{p}+0,05)$.

La sedación posterior a la infusión de dexmedetomidina no demostró significancia estadística en comparación con el grupo de lidocaína ( $\mathrm{p}+0,05)$.

\section{DISCUSIÓN}

Según sólo la definición, el dolor refractario es una condición de urgencia en donde el paciente, aún con tratamiento analgésico agresivo, no logra un control adecuado del dolor, por lo que la búsqueda de tratamiento efectivo y eficaz es prioritaria.

En la literatura existen demostraciones de la eficacia de la dexmedetomidina en pacientes con dolor refractario, aunque sólo han sido reportes de casos $(13,14)$; aún así se confirma lo observado en estos estudios, la dexmedetomidina prolonga el tiempo de analgesia sin presencia de efectos indeseables como hipotensión o disminución de frecuencia respiratoria y sin una disminución significativa en frecuencia cardiaca.

La lidocaína para dolor neuropático ha sido estudiada en múltiples ocasiones; en algunos estudios se ha observado una duración variable de efecto analgésico que va desde unas pocas horas a semanas después de una sola infusión i.v. $(18,19)$. Así mismo se ha encontrado una duración media de alivio del dolor de 9,34 días después de la infusión única, que va mucho más allá del periodo de infusión (80 minutos) o de la vida media del fármaco en plasma (60 a 90 minutos). Se han encontrado buenos resultados y sin presencia de riesgo con infusiones de $5 \mathrm{mg} / \mathrm{kg}$ de lidocaína durante 60 minutos (20); $5 \mathrm{mg} / \mathrm{kg}$ en 30 minutos (21); 2 y $5 \mathrm{mg} / \mathrm{kg}$, en 40 minutos (22), $500 \mathrm{mg}$ de lidocaína durante 60 minutos (23) y hasta $1,5 \mathrm{mg} /$ $\mathrm{kg}$ de lidocaína en un minuto. Esto es similar a lo encontrado en este estudio en donde con $3 \mathrm{mg} / \mathrm{kg}$ se logró una adecuada analgesia entre 40 minutos llegando incluso a 48 horas.

Hasta ahora no se han reportado estudios que comparen la dexmedetomidina y la lidocaína para dolor refractario a opioides en población paliativa.

El estudio puede ser aplicable a pacientes mayores de 18 años que presenten dolor refractario a opioides en cuidados paliativos. El estudio se realizó en población latina sin integrar otras etnias.

Una desventaja del estudio fue el no poder medir de manera exacta las concentraciones plasmáticas de los medicamentos para su comparación.

\section{CONCLUSIONES}

La analgesia resultante con ambas infusiones es similar durante el tiempo que se mantiene en infusión y en la postinfusión.
El número de rescates fue mayor en el grupo con lidocaína al ser requeridos en 5 ocasiones ( 3 durante la infusión y 2 postinfusión) a comparación del grupo de dexmedetomidina en donde fue requerido 1 rescate postinfusión.

El $28 \%$ del grupo de lidocaína no fue incluido en el muestreo postinfusión por no presentar un adecuado control algológico (EVA 6 o mayor); el $100 \%$ de los pacientes del grupo de la dexmedetomidina presentó un adecuado control después de los 60 minutos de infusión.

Se reportó mayor sedación con la infusión de dexmedetomidina durante la infusión sin presencia de complicaciones cardiovasculares y/o respiratorias ni durante ni posteriores a la infusión.

CORRESPONDENCIA:

Orlando Carrillo Torres

Servicio Clínica del Dolor

Hospital General de México O.D.

e-mail: orlo_78@hotmail.com

\section{BIBLIOGRAFÍA}

1. Estrategia Nacional de Cuidados Paliativos del Sistema Nacional de Salud. Ministerio de Sanidad y Consumo. España, 2007.

2. Shekhar S, Rajagopal M, Gayatri P, Charu S, Altaf GH. A phase II Pilot study to evaluate use of intraveous lidocaine for opioid refractarory pain in cancer patients. J Pain Symptom Manage 2009;3(1):85-93.

3. Merskey H. Classification of chronic pain. Descriptions of chronic pain syndromes and definitions of pain terms. Pain 1986;3(1).

4. Nazarian A, Christianson CA, Hua XY, Yaksh TL. Dexmedetomidine and ST-91 analgesia in the formalin model is mediated by $2 \mathrm{~A}$-adrenoceptors. Br J Pharmacol 2008;155:1117-26.

5. Grosu I, Lavand'homme P. Use of Dexmedetomidine for pain control. F 1000 Med Rep [serial on the Internet] 2010.

6. Eisenach J. Preemptive hyperalgesia, not analgesia? Anesthesiology [Editorial]. 2000;92(2):308-9.

7. Klimscha W, Chiari A, Krafft P, Plattner O, Taslimi R, Mayer N, et al. Hemodynamic and analgesic effects of clonidine added repetitively to continuous epidural and spinal blocks. Anesth Analg 1995;80:322-7.

8. Curatolo M, Petersen-Felix S, Arendt-Nielsen L, Zbinden AM. Epidural epinephrine and clonidine: Segmental analgesia and effects on different pain modalities. Anesthesiology 1997;87(4):785-94.

9. Wienbroun A, Ben AR. Dextromethorphan and dexmedetomidine, new agents for the control of perioperative pain. Eur J Surg 2001;167:563-9.

10. Hayashid K, Eisenach JC. Spinal_2 adrenoreceptor-mediated analgesia in neurophatic pain reflex brain-derived nerve growth factor and changes in spinal cholinergic neuronal function. Anesthesiology 2010;113:406-12.

11. Puke M, Wiesenfeld-Hallin Z. The differential effects of morphine and the a-adrenoceptor agonists clonidine and 
dexmedetomide on the prevention and treatment of experimental neurophatic pain. Anesth Analg 1993;77:104-9.

12. Roberts S, Wozencraft CP, Coyne PJ, Smith TJ. Dexmedetomidine as an adjuvant analgesic for intractable cancer pain. J Palliat Med 2011;14(3):371-3.

13. Prommer E. Dexmedetomidine: Do it have potential in palliative medicine? American Journal of Hospice and Palliative Medicine 2011;28(4).

14. Coyne P, Wozencraft CP, Roberts SB, Barton B, Smith TJ. Dexmedetomidine: Exploring its potential role and dosing guideline for its use in intractable pain in the palliative care setting. J Pain Palliat Care Pharmacother 2010;24(4):384-6.

15. Challapalli V, Tremont-Lukats IW, McNicol ED, Lau J, Carr DB. Administración sistémica de Agentes anestésicos locales para aliviar el dolor neuropático 2008;(2).

16. Whizar-Lugo V, Ochoa G. Conceptos actuales en dolor por cáncer. Anestesia en México 2005;17(Supl. 1).

17. Bach F, Jensen TS, Kastrup J, Strigsby B, Dejgard A. The effect of intravenous lidocaine on nociceptive processing in diabetic neuropathy. Pain 1990;40:29-34.
18. Kvarnström A, Karlsten R, Quiding H, Gordh T. The analgesic effect of intravenous ketamine and lidocaine on pain after spinal cord injury. Acta Anaesthesiol Scand 2004;48(4):498-506.

19. Ellemann K, Sjögren P, Banning AM, Jensen TS, Smith T, Geertsen P. Trial of intravenous lidocaine on painful neuropathy in cancer patients. Clin J Pain 1989;5(4):291-4.

20. Khan A, Burad J, Mehta C. Intravenous lidocaine infusion for neuropathic pain in cancer patients: A preliminary study. Indian J Anaesth 2002;46(5):360-4.

21. Kastrup J, Petersen P, Dejgard A, Angelo HR, Hilsted J. Intravenous lidocaine infusion new treatment of chronic painful diabetic neuropathy. Pain 1987;28:69-75.

22. Ferrante F, Paggioli J, Cherukuri S, Arthur GR. The analgesic response to intravenous lidocaine in the treatment of neuropathic pain. Anesth Analg 1996;82(1)91-7.

23. Galer B, Miller KV, Rowbotham MC. Response to intravenous lidocaine infusion differs based on clinical diagnosis and site of nervous system injury. Neurology $1993 ; 43(6): 1233-5$. 